\title{
Epigenetic regulation of RUNX3 in systemic sclerosis pathogenesis: time to target?
}

\author{
Steven O'Reilly
}

Systemic sclerosis (SSc) is an idiopathic autoimmune disease, that is, characterised by inflammation, cytokine imbalances and vasculopathy that leads to skin and lung fibrosis. The disease has very limited options with none specifically targeting the fibrotic component, and only in recent times has an insight into the molecular pathways at work come to light. An aberrant immune system has long been associated with SSc, with clear alterations in innate immune cell numbers and activation and recently the role of type I interferons has been implicated in SSc, ${ }^{1}$ molecules normally associated with a different rheumatic disease: systemic lupus erythematosus. What is driving this increase in type I interferons, however, was unknown until now.

In this issue, Affandi et al show that plasmacytoid dendritic cells (pDCs) are important and the central transcription factor RUNX3 is critical. ${ }^{2}$ The authors showed that RUNX3 expression is severely reduced in SSc patients, in pDCs, and this is observed across both limited and diffuse subsets of the disease. The level of RUNX3 was reduced both at the gene and protein levels and, importantly, correlated significantly with the Rodnan skin score. pDCs are rare type I interferon-producing cells that can present antigen. These cells can release high levels of type I interferon and can also contribute to pathology by the activation of effector $\mathrm{T}$ cells, cytotoxic $\mathrm{T}$ cells and conventional dendritic cells (DCs). Thus, it is conceivable that the pDCs in SSc are contributing to the disease pathology through the release of interferon and the activation of $\mathrm{T}$ cells, which are known to release profibrotic molecules capable of activating fibroblasts to myofibroblasts. ${ }^{3}$ RUNX3 is one of three mammalian Runt-domain transcription factors encoded by the highly conserved RUNX gene family. RUNX genes are key regulators of lineage-specific gene expression and regulate $\mathrm{T}$ cell development and RUNX3 is highly expressed

Correspondence to Dr Steven O'Reilly, Applied Sciences, Northumbria University, Newcastle upon Tyne NE1 8ST, UK; steven.oreilly@northumbria.ac.uk in conventional DC where it functions as part of the transforming growth factor- $\beta$ signalling pathway. Interestingly, genetic loss of RUNX3 in mice leads to spontaneous eosinophilic airway inflammation and airway remodelling including increased collagen deposition, which is suggestive of fibrosis. ${ }^{4}$ Interestingly, DC-specific RUNX3 knock out (KO) cells display and enhanced maturation and potency to stimulate T cells. ${ }^{4}$ While the role of RUNX3 in conventional DCs has been confirmed, ${ }^{4}$ its role in pDCs is highly novel. A small nucleotide polymorphism in RUNX3 has been associated with ankylosing spondylitis (AS) risk and that peripheral blood mononuclear cells have reduced expression of RUNX3 too. ${ }^{5}$ Interestingly, in AS cells containing the polymorphism this appears to contain the binding of transcription factors including P300. ${ }^{5}$ P300 is a transcriptional coactivator and acetyltransferase that mediates acetylation of histone tails leading to modulation of transcription and therefore, gene expression. It is known that $\mathrm{P} 300$ is important in SSc pathogenesis ${ }^{6}$ and it could be that alterations in RUNX3 mediate changes in expression of the acetyltransferase P300 leading to the opening of chromatin and subsequent gene expression in SSc pDCs.

In search of what is causing the reduced expression of RUNX3, the authors examined the methylation status of the gene for RUNX3 and found that within specific 5'-C-Phosphate-G-3' (CpG) sites that this was hypermethylated compared with healthy controls. Methylation of specific $\mathrm{CpG}$ sites within genes leads to gene repression through altering the binding of DNA. Conversely, hypomethylation leads to increased gene expression. ${ }^{6}$ The addition of methyl groups is controlled by the enzymes DNA methyl transferases and the removal by ten eleven translocation (TET) proteins. To confirm a direct role for methylation in controlling RUNX3 expression, the authors used the DNA demethylating agent 5-aza-2'-deoxycytidine and confirmed that incubation of the pan demethylating agent increased expression of RUNX3 in SSc cells. This suggests that the therapeutic use of 5 -aza-2'-deoxycytidine could be possible in SSc. Indeed decitabine (Dacogen), which is 5 -aza-2'-deoxycytidine, is already licenced for myleodysplastic syndrome and appears well tolerated, with few side effects. We have previously found that incubation of SSc dermal fibroblasts with decitabine reduced excess collagen production from such cells. $^{7}$

Interestingly, the authors also found that hypoxia also reduced the expression of RUNX3 and that hypoxia-induced reduction of RUNX3 could be blocked by small-interfering RNA to the central transcription factors hypoxia-inducible factors (HIF) 1 and 2- $\alpha$. Underscoring the role of the HIFs in mediating the effects of hypoxia in diminishing RUNX3 expression. It is of note that hypoxia has been reported in the skin and hands of SSc patients and that this may be an initial triggering factor that sets of the reduction of RUNX3 via increased methylation of the promoter. Indeed, hypoxia itself is critical in mediating activation of myofibroblasts in SSc. ${ }^{8}$ What the authors do not demonstrate is if hypoxia itself is directly regulating DNA methylation of the RUNX3 promoter. This would have been an interesting observation and could have been mediated by an increase in the expression and/or activity of DNA methyltransferase3a (DNMT3a) enzymes. This could also have been mediated be a decrease in the removal of DNA methyl marks which is mediated by the TET family of enzymes. Indeed, other epigenetic changes could have been mediated by hypoxia including a decrease of DNA methyltransferase (DNMT)-targeting microRNAs, thus leading to enhanced methylation at RUNX3 promoter, through enhanced DNMTs. Furthermore, the specific methylation site within RUNX3 could possibly serve as a marker of treatment response; that is, the methylation status could be used to guide response to treatment. Currently, there is no accepted biomarker for SSc.

The authors go on to show that RUNX3 is significantly downregulated in SSc skin tissue in situ and interestingly in cells other than pDC. Thus, cells other than pDC could also be contributing to the pathology. In mouse, bone marrow-derived $\mathrm{pDCs}$ that are specifically deleted for RUNX3 compared with matched RUNX3 $3^{\mathrm{f} / \mathrm{f}}$ cells stimulation with the tolllike receptor agonist $\mathrm{CpG}$ led to increased levels of CD40, CD80, CD86 and reduced CD62L. These are surface markers associated with increased maturation. Using 
the Cre-Lox system and a standard bleomycin model of fibrosis, where subcutaneous injection of bleomycin leads to skin fibrosis. It was found that mice deficient in RUNX3 had exaggerated fibrosis, with increased myofibroblasts and connective tissue growth factor and tissue inhibitor of metalloproteinases-1 (TIMP-1) levels. Interestingly, Siglec-H + pDCs were also higher in the skin of RUNX3 KO mice, which were drastically increased on exposure to bleomycin. Siglec $\mathrm{H}$ is a murine plasmacytoid-specific cell surface receptor, that is, part of the sialic acid-binding Ig-like lectin family that if blocked by a Siglec $\mathrm{H}$-specific antibody reduces interferon levels. Examination of the lymph nodes revealed significantly elevated frequencies of CD68 + DCs in RUNX3-specific KO cells on bleomycin injection; this indicates an elevated activation phenotype primed to trigger an adaptive immune response in the very area responsible for this. The antigen presentation ability of the pDCs devoid of RUNX3 in the lymph nodes would have been a useful experiment to do, determining their presenting ability to $\mathrm{T}$ cells.

The work presented here demonstrates that RUNX3 is a critical regulator of pDCs and that this is modulated by epigenetic regulation. Hypermethylation is present in RUNX3 at specific CpG sites and incubation of these cells with decitabine restored the levels of RUNX3. Functionally, the cells stimulated with a toll-like receptor (TLR) agonist in RUNX3 KOs displayed an activated mature phenotype with higher expression of cell surface markers indicative of an activated phenotype that could help activate naïve T cells. It is known that $\mathrm{T}$ cells themselves, especially a T-helper 2 polarised phenotype can drive the activation of local fibroblasts to secrete copious amounts of collagen via cytokines, including IL-4 and IL-13. ${ }^{3}$

The authors here do not directly investigate this hypothesis but it is worthy of further investigation in the future. pDCs produce high levels of interferon in response to activation of TLR7 and TLR9.
The authors use a synthetic ligand to stimulate their cells in vitro to measure their response across the two genotypes in terms of maturation and interferon production, seeing large changes. However, the actual DNA or RNA ligand in vivo in patients is yet unidentified. No viral aetiology in SSc is currently accepted.

We have previously demonstrated that TLR8 activation is profibrotic in SSc using both a synthetic commercially available ligand which is single-stranded RNA, but importantly serum isolated from patients could also trigger this, in a myeloid differentiation primary response 88 (MyD88)-nuclear factor kappalight-chain enhancer of activated $\mathrm{B}$ cells (NF-KB)-dependent manner. ${ }^{9} \quad$ Furthermore, this serum-mediated TLR8 effect could be reduced by incubation with an RNAse enzyme which degrades RNA.

Thus, it is suggested that extracellular RNA, possibly released from dead cells in the vasculature, may trigger a TLR response in pDCs, that leads to increased maturation and activation of $\mathrm{T}$ cells leading to a panoply of profibrotic cytokines activating resident fibroblasts. This requires further investigation but would be akin to the situation in systemic lupus erythematosus (SLE) where extracellular nucleic acid triggers autoimmunity. Given the key role of epigenetics and methylation in particular plays, use of a hypomethylating agent such as decitabine appears warranted. Finally, in SSc skin and lung biopsies, it was demonstrated that RUNX3 is reduced both in pDCs but other cells also not identified by any specific cell markers, given the known role of RUNX3 in T cell development, and the $\mathrm{T}$ cell dysfunction in SSc, I suggest that RUNX3 is epigenetically repressed here. This could render these cells pathogenic. The work presented adds an intriguing piece of the puzzle and suggests a critical role of innate immunity and epigenetics in SSc pathogenesis.

Handling editor Josef S Smolen

Contributors SO'R contributed to this article alone.
Funding The authors have not declared a specific grant for this research from any funding agency in the public, commercial or not-for-profit sectors.

Competing interests None declared.

Patient consent for publication Not required.

Provenance and peer review Commissioned; internally peer reviewed.

(c) Author(s) (or their employer(s)) 2019. No commercial re-use. See rights and permissions. Published by BMJ.

\section{Check for updates}

To cite O'Reilly S. Ann Rheum Dis

2019;78:1149-1150.

Received 15 May 2019

Revised 20 May 2019

Accepted 21 May 2019

Published Online First 29 May 2019

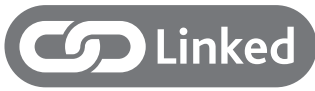

http://dx.doi.org/10.1136/annrheumdis-2018214991

Ann Rheum Dis 2019:78:1149-1150.

doi:10.1136/annrheumdis-2019-215648

\section{REFERENCES}

1 Fullard N, O'Reilly S. Role of innate immune system in systemic sclerosis. Semin Immunopathol 2015;37:511-7.

2 Affandi AJ, Carvalheiro T, Ottria A, et al. Low RUNX3 expression alters dendritic cell function in patients with systemic sclerosis and contributes to enhanced fibrosis. Ann Rheum Dis 2019;78:1249-59.

3 O'Reilly S, Hügle T, van Laar JM. T cells in systemic sclerosis: a reappraisal. Rheumatology 2012;51:1540-9.

4 Fainaru 0 , Woolf E, Lotem J, et al. Runx3 regulates mouse TGF-beta-mediated dendritic cell function and its absence results in airway inflammation. Embo J 2004:23:969-79.

5 Vecellio M, Cortes A, Roberts AR, et al. Evidence for a second ankylosing spondylitis-associated $R U N X 3$ regulatory polymorphism. RMD Open 2018;4:e000628.

6 Henderson J, Distler J, O'Reilly S. The role of epigenetic modifications in systemic sclerosis: a druggable target. Trends Mol Med 2019;25:395-411.

7 O'Reilly S, Ciechomska M, Fullard N, et al. IL-13 mediates collagen deposition via STAT6 and microRNA135b: a role for epigenetics. Sci Rep 2016;6.

8 Distler JHW, Jüngel A, Pileckyte M, et al. Hypoxiainduced increase in the production of extracellular matrix proteins in systemic sclerosis. Arthritis Rheum 2007:56:4203-15.

9 Ciechomska M, Huigens CA, Hügle T, et al. Toll-like receptor-mediated, enhanced production of profibrotic TIMP-1 in monocytes from patients with systemic sclerosis: role of serum factors. Ann Rheum Dis 2013;72:1382-9. 